\title{
Formal Verification of SSA-Based Optimizations for LLVM
}

\author{
Jianzhou Zhao Santosh Nagarakatte ${ }^{\dagger} \quad$ Milo M. K. Martin Steve Zdancewic \\ Computer and Information Science Department, University of Pennsylvania ${ }^{\dagger}$ Rutgers University \\ jianzhou@cis.upenn.edu santosh.nagarakatte@cs.rutgers.edu milom@cis.upenn.edu stevez@cis.upenn.edu
}

\begin{abstract}
Modern compilers, such as LLVM and GCC, use a static single assignment (SSA) intermediate representation (IR) to simplify and enable many advanced optimizations. However, formally verifying the correctness of SSA-based optimizations is challenging because SSA properties depend on a function's entire control-flow graph.

This paper addresses this challenge by developing a proof technique for proving SSA-based program invariants and compiler optimizations. We use this technique in the Coq proof assistant to create mechanized correctness proofs of several "micro" transformations that form the building blocks for larger SSA optimizations. To demonstrate the utility of this approach, we formally verify a variant of LLVM's mem2reg transformation in Vellvm, a Coq-based formal semantics of the LLVM IR. The extracted implementation generates code with performance comparable to that of LLVM's unverified implementation.
\end{abstract}

Categories and Subject Descriptors D.2.4 [Software Engineering]: Software/Program Verification - Correctness Proofs; F.3.1 [Logics and Meanings of Programs]: Specifying and Verifying and Reasoning about Programs - Mechanical verification; F.3.2 [ $\mathrm{Log}_{-}$ ics and Meanings of Programs]: Semantics of Programming Languages - Operational semantics

General Terms Languages, Verification, Reliability

Keywords LLVM, Coq, single static assignment

\section{Introduction}

Compiler bugs can manifest as crashes during compilation, or, much worse, result in the silent generation of incorrect programs. Such mis-compilations can introduce subtle errors that are difficult to diagnose and generally puzzling to software developers. A recent study by Yang et al. [20] used random test-case generation to expose serious bugs in mainstream compilers including GCC, LLVM, and commercial compilers. Whereas few bugs were found in the front end of the compiler, various optimization phases of the compiler that aim to make generated programs faster were a prominent source of bugs.

Projects like CompCert [9, 16-18] are tackling the problem of compiler bugs by mechanically verifying the correctness of compilers. Indeed, although the aforementioned study uncovered many bugs in other compilers, the only bugs found in CompCert were in those parts of the compiler not (yet) formally verified. Yang et al. write [20]: "The apparent unbreakability of CompCert supports

Permission to make digital or hard copies of all or part of this work for personal or classroom use is granted without fee provided that copies are not made or distributed for profit or commercial advantage and that copies bear this notice and the full citation on the first page. To copy otherwise, to republish, to post on servers or to redistribute to lists, requires prior specific permission and/or a fee.

PLDI'13, June 16-19, 2013, Seattle, WA, USA.

Copyright (C) 2013 ACM 978-1-4503-2014-6/13/06. . \$15.00 a strong argument that developing compiler optimizations within a proof framework, where safety checks are explicit and machinechecked, has tangible benefits for compiler users."

Despite CompCert's groundbreaking compiler-verification efforts, there still remain many challenges in applying its technology to industrial-strength compilers. In particular, the original CompCert development, and the bulk of the subsequent work-with the notable exception of CompCertSSA [4] — did not use a static single assignment (SSA) [7] intermediate representation (IR) [9].

In SSA intermediate representations, each variable of a function is assigned statically only once, and each variable definition must dominate all of its uses in the control-flow graph. These SSA properties simplify or enable many compiler optimizations [14], including: constant folding, sparse conditional constant propagation, aggressive dead code elimination, global value numbering, global code motion, partial redundancy elimination, and inductive variable analysis. Consequently, open-source and commercial compilers such as GCC, LLVM, and Intel CC all use SSA-based intermediate representations when performing such optimizations.

Despite their importance, there are few mechanized formalizations of the correctness properties of SSA transformations. This paper tackles this problem by developing proof techniques suitable for mechanically verifying the correctness of SSA-based optimizations. We do so in the context of our Vellvm framework [21, 22], which formalizes the operational semantics of programs expressed in LLVM's SSA-based IR [10] and provides Coq [6] infrastructure to facilitate mechanized proofs of properties about transformations on the LLVM IR.

The key idea is to generalize the "SSA scoping" predicate, which is an invariant of the operational semantics, to more complex safety predicates. The main proof obligation then becomes a preservation argument, but one that takes the dominance relation of the SSA form into account. Instances of this idea are found in the literature (see, for example, Menon, et al. [13]), and related proof techniques have been recently used in CompCertSSA [4], which uses translation validation to check SSA optimizations (see Section 8). This work is the first to verify SSA algorithms in the context of an industrial-strength compiler like the LLVM.

To demonstrate the utility of these techniques, we use them to establish the correctness of a variant of mem 2 reg, a crucial LLVM pass that is responsible for promoting un-aliased local variables and stack-based temporary values into registers. We build our vmem 2 reg pass by "pipelining" several simpler transformations, such as load-after-store elimination, which are common building blocks of many SSA-based optimizations. To improve compilation time, we also investigate how to fuse such multiple such passes into one. To summarize our contributions, this paper:

- introduces general-purpose proof techniques for verifying SSA optimizations,

- shows that the proposed SSA proof techniques are viable for use in mechanized proofs, and verifies several simple transformations, 
- describes and proves correct vmem2reg, an easier-to-verify variant of LLVM's mem2reg optimization, which is a key SSA-based transformation used by LLVM, and

- demonstrates that the extracted implementation of the fully verified vmem2reg is effective: The vmem2reg fully-verified pass yields an average speedup over a LLVM -00 of 77\% versus a speedup of $81 \%$ speedup obtained by LLVM's implementation of mem2reg.

To streamline the explanation (Section 3), we describe the proof techniques in the context of Vminus, a simplified subset the full Vellvm framework [22], but one that still captures the essence of SSA. We mechanically verified all the claims of the paper both for Vminus and full Vellvm in Coq. ${ }^{1}$

\section{Background}

\subsection{Program refinement}

In this paper, we use program refinement to reason about the correctness of compilation. Following the CompCert project [9], we define program refinement in terms of programs' external behaviors (which include program traces of input-output events, whether a program terminates, and the returned value if a program terminates): a transformed program refines the original if the behaviors of the original program include all the behaviors of the transformed program. We define the operational semantics using traces of a labeled transition system.

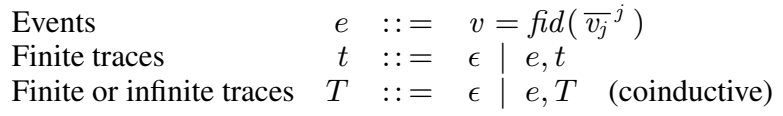

We denote one small-step of evaluation as config $\vdash S \stackrel{t}{\longrightarrow} S^{\prime}$ : in program environment config, program state $S$ transitions to the state $S^{\prime}$, recording events $e$ of the transition in the trace $t$. An event $e$ describes the inputs $v_{j}$ and output $v$ of an external function call named fid. config $\vdash S \stackrel{t}{\longrightarrow} S^{\prime}$ denotes the reflexive, transitive closure of the small-step evaluation with a finite trace $t$. config $\vdash S \stackrel{T}{\longrightarrow} \infty$ denotes a diverging evaluation starting from $S$ with a finite or infinite trace $T$. Program refinement is given by the following definition.

DEFinition 1 (Program refinement).

1. init ( prog, fid, ${\overline{v_{j}}}^{j}, S$ ) means $S$ is the initial program state of the program prog with the main entry fid and inputs $v_{j}$.

2. final $(S, v)$ means $S$ is the final state with the return value $v$.

3. $\Downarrow\left(\right.$ prog, fid $\left.,{\overline{v_{j}}}^{j}, t, v\right)$ means $\exists S S^{\prime}$. init (prog, fid $\left.,{\overline{v_{j}}}^{j}, S\right)$, config $\vdash S \stackrel{t}{\longrightarrow} S^{\prime}$ and final $\left(S^{\prime}, v\right)$.

4. $\left(\right.$ prog, fid, ${\overline{v_{j}}}^{j}, T)$ means $\exists S$. init (prog, fid $\left.,{\overline{v_{j}}}^{j}, S\right)$ and config $\vdash S \stackrel{T}{\longrightarrow} \infty$.

\left. 5. \& (prog, fid, ${\overline{v_{j}}}^{j}, t\right)$ means $\exists S S^{\prime}$. init (prog, fid, ${\overline{v_{j}}}^{j}, S)$, config $\vdash S \stackrel{t}{\longrightarrow} S^{\prime}$ and $S^{\prime}$ is stuck.

\left. 6. defined (prog, fid, ${\overline{v_{j}}}^{j}\right)$ means $\forall t$, $\neg \Downarrow\left(\right.$ prog $\left., f i d,{\overline{v_{j}}}^{j}, t\right)$

7. prog $_{2}$ refines program $\operatorname{prog}_{1}$, written $\operatorname{prog}_{1} \supseteq \operatorname{prog}_{2}$, if

(a) defined $\left(\operatorname{prog}_{1}, f i d,{\overline{v_{j}}}^{j}\right)$

(b) $\Downarrow\left(\operatorname{prog}_{2}, f i d,{\overline{v_{j}}}^{j}, t, v\right) \Rightarrow \Downarrow\left(\operatorname{prog}_{1}, f i d,{\overline{v_{j}}}^{j}, t, v\right)$

(c) $\Uparrow\left(\operatorname{prog}_{2}, f i d, \bar{v}_{j}^{j}, T\right) \Rightarrow \Uparrow\left(\operatorname{prog}_{1}, f i d,{\overline{v_{j}}}^{j}, T\right)$

$(d) \quad \forall\left(\operatorname{prog}_{2}, f i d,{\overline{v_{j}}}^{j}, t\right) \Rightarrow \forall\left(\operatorname{prog}_{1}, f i d,{\overline{v_{j}}}^{j}, t\right)$

Note that refinement requires only that a transformed program preserves the semantics of a well-defined original program, but does not constrain the transformation of undefined programs.

\footnotetext{
${ }^{1}$ Annotated Coq source available at http://cis.upenn.edu/ stevez/vellvm/release.tgz.
}

\begin{tabular}{|c|c|c|}
\hline Lock-step & Right "option" & Left "option" \\
\hline $\mathrm{S}_{1} \sim \mathrm{S}_{2}$ & $\mathrm{~S}_{1} \sim \mathrm{S}_{2}$ & $\mathrm{~S}_{1} \sim \mathrm{S}_{2}$ \\
\hline & $\mathrm{t}$ or $\epsilon$ & $\mathrm{t}$ or \\
\hline $\mathrm{I}_{1}^{-\sim} \sim-\mathrm{S}_{2}^{\prime}$ & $\begin{array}{l}\mathrm{S}_{1^{-}-\mathrm{S}_{2}} \quad \mathrm{~S}_{1_{1}^{\prime}}^{\prime} \\
\quad \text { (with }\left|\mathrm{S}_{1}^{\prime}\right|<\left|\mathrm{S}_{1}\right| \text { ) }\end{array}$ & 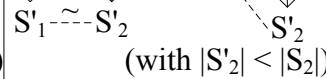 \\
\hline
\end{tabular}

Figure 1. Backward simulation diagrams that imply program refinement. In each diagram, the program states of original and compiled programs are on the left and right respectively. A line denotes a relation $\sim$ between program states. Solid lines or arrows denote hypotheses; dashed lines or arrows denote conclusions.

We use the backward simulation diagrams in Figure 1 to prove that a program transformation satisfies the refinement property. The CompCert project uses similar diagrams for forward simulation [9]. At a high-level, we first need to find a relation $\sim$ between program states and their transformed counterparts. The relation must hold initially, imply equivalent returned values finally, and imply that stuck states are related. Then, depending on the transformation, we prove that a specific diagram holds: lock-step simulation is for variable substitution, right "option" simulation is for instruction removal, and left "option" simulation is for instruction insertion. Because the existence of a diagram implies that the source and target programs share traces, we can prove the equivalence of program traces by decomposing program transitions into matched diagrams. To ensure co-termination, the "option" simulations are parameterized by a measure of program states $|S|$ that must decrease to prevent "infinite stuttering" problems.

\subsection{SSA}

Static Single Assignment (SSA) form [7] is an intermediate representation distinguished by its treatment of temporary variableseach such variable may be defined only once, statically, and each use of the variable must be dominated by its definition with respect to the control-flow graph of the containing function. ${ }^{2}$ Intuitively, the variable definition dominates a use if all possible execution paths to the use go through the definition first.

To maintain these invariants in the presence of branches and loops, SSA form uses $\phi$-instructions, which act like control-flow dependent move operations. Such $\phi$-instructions appear only at the start of a basic block and, crucially, they are handled specially in the dominance relation to "cut" apparently cyclic data dependencies.

The left part of Figure 2 shows an example program in SSA form, written using the stripped-down notation of Vminus (defined more formally in Section 3). The temporary $r_{3}$ at the beginning of the block labeled $l_{2}$ is defined by a $\phi$-instruction: if control enters the block $l_{2}$ by jumping from basic block $l_{1}, r_{3}$ will get the value 0 ; if control enters from block $l_{2}$ (via the back edge of the branch at the end of the block), then $r_{3}$ will get the value of $r_{5}$.

The SSA form is good for implementing optimizations because it identifies variable names with the program points at which they are defined. Maintaining the SSA invariants thus makes definition and use information of each variable more explicit. Also, because each variable is defined only once, there is less mutable state to be considered (for purposes of aliasing, etc.) in SSA form, which makes certain code transformations easier to implement.

Program transformations like the one in Figure 2 are correct if the transformed program refines the original program (in the sense described above) and the result is well-formed SSA. Proving that

\footnotetext{
2 In the literature, there are different variants of SSA forms [1]. We use LLVM' version: memory locations are not in SSA form; LLVM does not maintain any connection between a variable name in the IR and its original name in the source; the live ranges of variables can overlap.
} 


\begin{tabular}{cc}
\hline Original & Transformed \\
\hline & \\
$l_{1}: \ldots$ & $l_{1}: \cdots$ \\
$\ldots$ & $r_{4}:=r_{1} * r_{2}$ \\
$\mathbf{b r} r_{0} l_{2} l_{3}$ & $\mathbf{b r} r_{0} l_{2} l_{3}$ \\
$l_{2}: r_{3}=\mathbf{p h i \operatorname { i n t }}\left[0, l_{1}\right]\left[r_{5}, l_{2}\right]$ & $l_{2}: r_{3}=\mathbf{p h i i n t}\left[0, l_{1}\right]\left[r_{5}, l_{2}\right]$ \\
$r_{4}:=r_{1} * r_{2}$ & \\
$r_{5}:=r_{3}+r_{4}$ & $r_{5}:=r_{3}+r_{4}$ \\
$r_{6}:=r_{5} \geq 100$ & $r_{6}:=r_{5} \geq 100$ \\
$\mathbf{b r} r_{6} l_{2} l_{3}$ & $\mathbf{b r} r_{6} l_{2} l_{3}$ \\
$l_{3}: r_{7}=\mathbf{p h i \operatorname { i n t }}\left[0, l_{1}\right]\left[r_{5}, l_{2}\right]$ & $l_{3}: r_{7}=\mathbf{p h i i n t}\left[0, l_{1}\right]\left[r_{5}, l_{2}\right]$ \\
$r_{8}:=r_{1} * r_{2}$ & \\
$r_{9}:=r_{8}+r_{7}$ & $r_{9}:=r_{4}+r_{7}$
\end{tabular}

Figure 2. An SSA-based optimization. In the original program with entry $l_{1}, r_{1} * r_{2}$ is a partial common expression for the definitions of $r_{4}$ and $r_{8}$, because there is no domination relation between $r_{4}$ and $r_{8}$. Therefore, eliminating the common expression directly is not correct: we cannot simply replace $r_{8}:=r_{1} * r_{2}$ by $r_{8}:=r_{4}$ since $r_{4}$ is not available at the definition of $r_{8}$ if $l_{2}$ does not execute before $l_{3}$ runs. To transform this program, we might first move the instruction $r_{4}:=r_{1} * r_{2}$ from the block $l_{2}$ to the block $l_{1}$, because the definitions of $r_{1}$ and $r_{2}$ must dominate the end of $l_{1}$, and $l_{1}$ dominates $l_{2}$. Then we can safely replace all the uses of $r_{8}$ by $r_{4}$, because the definition of $r_{4}$ in $l_{1}$ dominates $l_{3}$ and therefore dominates all the uses of $r_{8}$. Finally, $r_{8}$ is removed, because there are no uses of $r_{8}$.

such code transformations are correct is nontrivial because they involve non-local reasoning about the program.

\section{Proof Techniques for SSA}

This section describes the proof techniques we have developed for formalizing properties of SSA-style intermediate representations. To most clearly articulate the approach, we present the results using a language called Vminus, which is a minimalist SSA language containing only those features salient to the proof technique. Vminus is a subset of the LLVM IR formalized in Vellvm [22].

The key idea of the technique is to generalize the invariant used for Vminus's preservation lemma for proving safety to other predicates that are also shown to be invariants of the operational semantics. Crucially, these predicates all share the same form, which only constrains variable definitions that strictly dominate the current program counter.

The remainder of this section first presents the syntax and operational semantics of Vminus, then it gives the static semantics and proves safety (which in this context simply amounts to showing that all variables are well-scoped). With these properties established, we then show how to generalize the safety invariant to a form that is useful for proving program transformations correct and demonstrate its applicability to a number of standard optimizations. Because Vminus is such a stripped-down language, the relevant lemmas are relatively straightforward to establish; Section 4 shows how to scale the proof technique to the full Vellvm model of LLVM to verify the mem2reg pass.

\subsection{The simple SSA language-Vminus}

Syntax Figure 3 gives the syntax of Vminus. Every Vminus expression is of type integer. Operations in Vminus compute with values $v a l$, which are either temporaries $r$ or constants $c n s t$ that must be integer values. We use $R$ to range over sets of identifiers.

All code in Vminus resides in a top-level function, whose body is composed of blocks $b$. Here, $\bar{b}$ denotes a list of blocks; we also use similar notation for other lists. As is standard, a basic block consists of a labeled entry point $l$, a series of $\phi$ nodes, a list of commands $c \mathrm{~s}$, and a terminator instruction tmn. In the following, we also use the label $l$ of a block to denote the block itself.

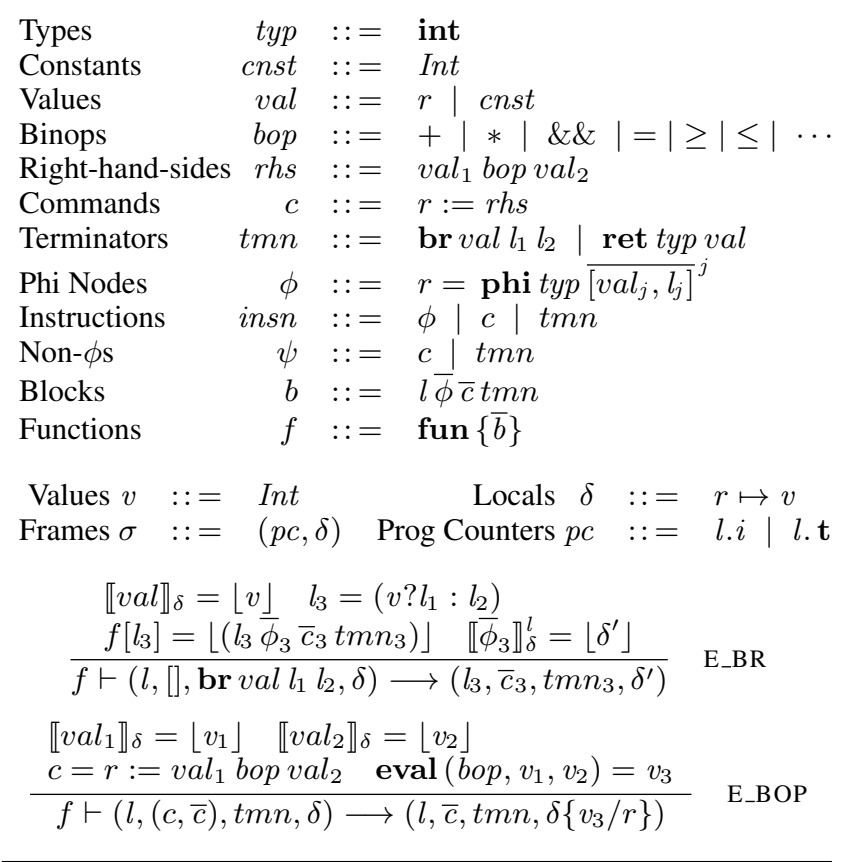

Figure 3. Syntax and Operational Semantics of Vminus

The set of blocks making up the top-level function constitutes a control-flow graph with a well-defined entry point that cannot be reached from other blocks. We write $f[l]=\lfloor b\rfloor$ if there is a block $b$ with label $l$ in function $f$. Here, the \lfloor\rfloor (pronounced "some") indicates that the function is partial (might return "none" instead).

As usual in SSA, the $\phi$ nodes join together values from a list of predecessor blocks of the control-flow graph-each $\phi$ node takes a list of (value, label) pairs that indicates the value chosen when control transfers from a predecessor block with the associated label. The commands $c$ include the usual suite of binary arithmetic or comparison operations. We denote the right-hand-sides of commands by rhs. Block terminators (br and ret) branch to another block or return a value from the function. We also use metavariable insn to range over $\phi$-nodes, commands and terminators, and non-phinodes $\psi$ to represent commands and terminators.

Dynamic Semantics The operational semantics rules in Figure 3 are parameterized by the top-level function $f$, and relate evaluation frames $\sigma$ before and after an evaluation step. An evaluation frame keeps track of the integer values $v$ bound to local temporaries $r$ in $\delta$ and current program counter. We also use $\sigma . p c$ and $\sigma . \delta$ to denote the program counter and locals of $\sigma$ respectively. Because Vminus has no function calls, the rules ignore program traces. This simplification does not affect the essence of the proof techniques. Section 5 shows the full Vellvm semantics with traces.

Instruction positions are denoted by program counters $p c: l . i$ indicates the $i$-th command in the block $l ; l$. t indicates the terminator of the block $l$. We write $f[p c]=\lfloor$ insn $\rfloor$ if some insn is at the program counter $p c$ of function $f$. We also use $l .(i+1)$ to denote the next program counter of $l . i$. When $l . i$ is the last command of block $l, l .(i+1)=l$.t. To simplify presentation of the operational semantics, we use $l, \bar{c}, t m n$ to "unpack" the instructions at a program counter in function $f$. Here, $l$ is the current block, $\bar{c}$ and $t m n$ are the instructions of $l$ that are not executed yet. It is easy to see how the "block \& offset" specification is equivalent to the "continuation commands" representation so we omit the details of the correspondence here. 


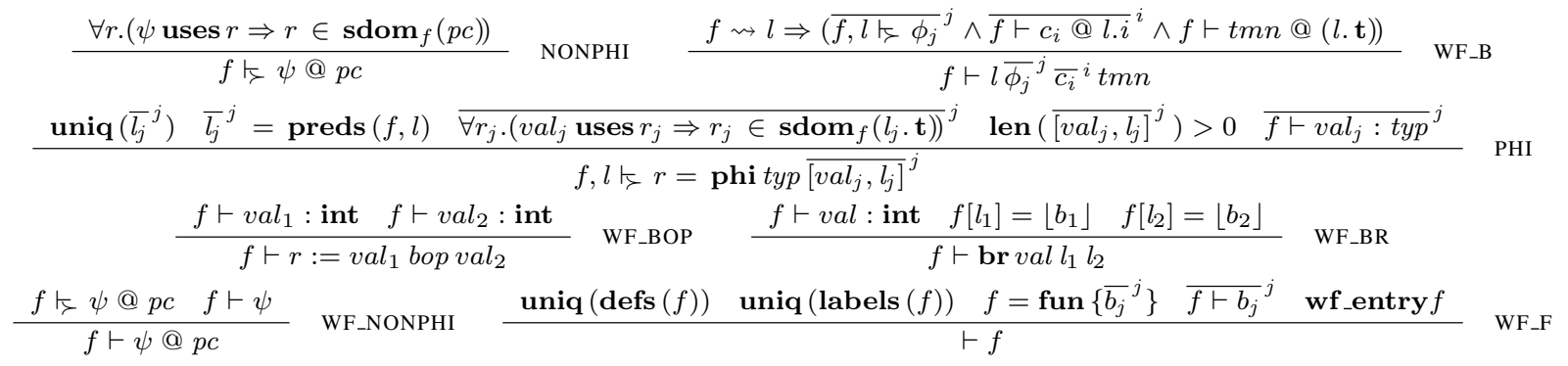

Figure 4. Static Semantics of Vminus (excerpt)

Most of the Vminus commands have straight-forward interpretation. The arithmetic and logic instructions are all unsurprisingthe $\llbracket v a l \rrbracket_{\delta}$ function computes a value from the local state $\delta$ and $v a l$, looking up the meanings of variables in the local state as needed; eval implements arithmetic and logic operations. We use $\llbracket r h s \rrbracket_{\delta}$ to denote evaluating the right-hand-side $r h s$ in the state $\delta$.

There is one wrinkle in specifying the operational semantics when compared to a standard environment-passing call-by-value language. All of the $\phi$ instructions for a block must be executed atomically and with respect to the "old" local value mapping due to the possibility of self loops and dependencies among the $\phi$ nodes. For example the well-formed code fragment bellow has a circular dependency between $r_{1}$ and $r_{2}$

$$
\begin{array}{ll}
l_{0}: & \cdots \\
l_{1}: & r_{1}=\text { phi int }\left[r_{2}, l_{1}\right]\left[0, l_{0}\right] \\
& r_{2}=\text { phi int }\left[r_{1}, l_{1}\right]\left[1, l_{0}\right] \\
& r_{3}:=r_{1}=r_{2} \\
& \text { br } r_{3} l_{2} l_{1} \\
l_{2}: & \cdots
\end{array}
$$

If control enters this block from $l_{0}, r_{1}$ will map to 0 and $r_{2}$ to 1 , which causes the conditional branch to fail, jumping back to the label $l_{1}$. The new values of $r_{1}$ and $r_{2}$ should be 1 and 0 , and not 1 and 1 as might be computed if they were handled sequentially. This atomic update of the local state, similar to "parallel assignment," is handled by the $\llbracket \bar{\phi}_{3} \rrbracket_{\delta}^{l}$ function as shown in rule E_BR.

Static semantics Vminus requires that a program satisfy certain invariants to be considered well formed: every variable in the toplevel function must dominate all its uses, and be assigned exactly, once statically. At a minimum, any reasonable Vminus transformation must preserve these invariants; together they imply that the program is in SSA form [7].

Figure 4 shows the judgments to check the SSA invariants with respect to the control-flow graph and program points of the function $f$. To explain the judgments we need the following definitions.

DEFINITION 2 (Domination).

1. val uses $r \triangleq v a l=r$.

2. insn uses $r \triangleq \exists$ val. val uses $r \wedge$ val is an operand of insn.

3. A variable $r$ is defined at a program counter pc of function $f$, written $f$ defines $r$ @ $p c$ if and only if $f[p c]=\lfloor$ insn $\rfloor$ and $r$ is the left-hand side of insn. We write defs $(f)$ to denote the set of all variables defined in $f$.

4. In function $f$, block $l_{1}$ dominates block $l_{2}$, written $f \vdash l_{1} \succcurlyeq l_{2}$, if every path from the entry block of $f$ to $l_{2}$ must go through $l_{1}$; $l_{1}$ strictly dominates $l_{2}$, written $f \vdash l_{1} \succ l_{2}$, if every path from the entry block of $f$ to $l_{2}$ must go through $l_{1}$ and $l_{1} \neq l_{2}$.

5. In function $f, p c_{1}$ strictly dominates $p c_{2}$, written $f \vdash p c_{1} \succ$ $p c_{2}$, if $p c_{1}$ and $p c_{2}$ are at distinct blocks $l_{1}$ and $l_{2}$ respectively and $f \vdash l_{1} \succ l_{2}$; if $p c_{1}$ and $p c_{2}$ are in the same block, then $p c_{1}$ appears earlier than $p c_{2}$.
6. $\operatorname{sdom}_{f}(p c)$ is the set of variables strictly dominating $p c$ :

$$
\operatorname{sdom}_{f}(p c)=\left\{r \mid f \text { defines } r @ p c^{\prime} \text { and } f \vdash p c^{\prime} \succ p c\right\}
$$

Rule WF_F of Figure 4 ensures that variables defs $(f)$ defined in the top-level function are unique, which enforces the singleassignment part of the SSA property. Additionally, all block labels labels $(f)$ in the function must be unique for a well-formed control-flow graph, and the entry block should have no predecessors (wf_entry $f$ ).

The rule WF_B checks that all instructions in reachable blocks (written $f \rightsquigarrow l$ ) satisfy the SSA domination invariant. Because unreachable blocks have no effects at runtime, the rule does not check them. Rule NONPHI ensures that a $\psi$ at $p c$ is strictly dominated by the definitions of all variables used by $\psi$. The rule PHI ensures that the number of incoming values is not zero, that all incoming labels are unique, and that the current block's predecessors is the same as the set of incoming labels. If an incoming value $\mathrm{val}_{j}$ from a predecessor block $l_{j}$ uses a variable $r_{j}$ at $p c_{j}$, then $p c_{j}$ must strictly dominate the terminator of $l_{j}$. Importantly, this rule allows "cyclic" uses of SSA variables of the kind shown in the example above.

Dominance analysis Dominance analysis plays an important role in the type system, so we must first prove the following lemmas about the domination relations. They are needed to establish the SSA-based program properties in the following sections. Details about these lemmas can be found our prior work [21].

LEMMA 1 (Domination is transitive).

LEMMA 2 (Strict domination is acyclic).

By Lemma 1, $\operatorname{sdom}_{f}(p c)$ has the following properties:

LEMMA 3 (sdom step).

1. If $l . i$ and $l .(i+1)$ are valid program counters of $f$, then $\operatorname{sdom}_{f}(l .(i+1))=\operatorname{sdom}_{f}(l . i) \cup\{r\}$ where $f$ defines $r @ l . i$.

2. If $l$. $\mathbf{t}$ and $l^{\prime} .0$ are valid program counters of $f$, and $l^{\prime}$ is a successor of $l$, then $\operatorname{sdom}_{f}\left(l^{\prime} .0\right)-\operatorname{defs}(\bar{\phi}) \subseteq \operatorname{sdom}_{f}(l . \mathbf{t})$ where $\bar{\phi}$ are from the block $l^{\prime}$ and $\operatorname{defs}(\bar{\phi})$ denotes all variables defined by $\bar{\phi}$.

\subsection{Safety of Vminus}

There are two ways that a Vminus program might get stuck. First, it might try to jump to an undefined label, but this property is ruled out statically by WF_BR. Second, it might try to access a variable whose value is not defined in $\delta$. We can prove that this second case never happens by establishing the following safety theorem:

THEOREM 4 (Safety). If $\vdash f$ and $f \vdash($ entry. $0, \emptyset) \longrightarrow^{*} \sigma$, then $\sigma$ is not stuck. 
The proof takes the standard form using preservation and progress lemmas with the invariant for frames shown bellow:

$$
\frac{p c \in f \quad \forall r .\left(r \in \mathbf{s d o m}_{f}(p c) \Rightarrow \exists v \cdot \delta[r]=\lfloor v\rfloor\right)}{f \vdash(p c, \delta)} \quad \text { WF_FR }
$$

This rule is similar to the predicate used in prior work for verifying the type safety of an SSA-based language [13]. The invariant WF_FR shows that a frame $(p c, \delta)$ is well-formed if every definition that strictly dominates $p c$ is defined in $\delta$. The initial program state satisfies this invariant trivially:

Lemma 5 (Initial State). If $\vdash f$ then $f \vdash($ entry.0, $\emptyset)$.

The preservation and progress lemmas are straightforward-but note that they crucially rely on the interplay between the invariant on $\delta$ "projected" onto $\operatorname{sdom}_{f}(p c)$ (Lemma 3), and the PHI and NONPHI rules of the static semantics.

Lemma 6 (Preservation). If $\vdash f, f \vdash \sigma$ and $f \vdash \sigma \longrightarrow \sigma^{\prime}$, then $f \vdash \sigma^{\prime}$.

LEMMA 7 (Progress). If $\vdash f, f \vdash \sigma$, then $\sigma$ is not stuck.

\subsection{Generalizing Safety to other SSA Invariants}

The main feature of the preservation proof, Lemma 6 , is that the constraint on $\operatorname{sdom}_{f}(p c)$ is an invariant of the operational semantics. But-and this is a key observation - we can parameterize rule WF_FR by a predicate $P$, which is an arbitrary proposition about functions and frames:

\begin{tabular}{|c|}
\hline$\sigma . p c \in f \quad P f\left(\left.\sigma\right|_{f}\right)$ \\
\hline$f, P \vdash \sigma$
\end{tabular} GWF_FR

Here, $\left.\sigma\right|_{f}$ is $\left(\sigma . p c,\left.(\sigma . \delta)\right|_{\left(\operatorname{sdom}_{f}(\sigma . p c)\right)}\right)$ and we write $\left(\left.\delta\right|_{R}\right)[r]=$ $\lfloor v\rfloor$ iff $r \in R$ and $\delta[r]=\lfloor v\rfloor$ and observe that $\operatorname{dom}\left(\left.\delta\right|_{R}\right)=R$. These restrictions say that we don't need to consider all variables: Intuitively, because SSA invariants are based on dominance properties, when reasoning about a program state we need only consider the variable definitions that strictly dominate the program counter in a given state.

For proving Theorem 4, we instantiated $P$ to be:

$$
P_{\text {safety }} \triangleq \lambda f . \lambda \sigma . \forall r . r \in \operatorname{dom}(\sigma . \delta) \Rightarrow \exists v \cdot(\sigma . \delta)[r]=\lfloor v\rfloor
$$

For safety, it is enough to show that each variable in the domination set is well defined at its use. To prove program transformations correct, we instantiate $P$ with a different predicate, $P_{\text {sem }}$, that relates the syntactic definition of a variable with the semantic value:

$\lambda f . \lambda \sigma . \forall r . f[r]=\lfloor r h s\rfloor \Rightarrow(\sigma . \delta)[r] \neq \cdot \Rightarrow(\sigma . \delta)[r]=\llbracket r h s \rrbracket_{(\sigma . \delta)}$

Just as we proved preservation for $P_{\text {safety }}$, we can also prove preservation for $P_{\text {sem }}$ (using Lemma 2):

THEOREM 8. If $\vdash f$ and $f, P_{\text {sem }} \vdash \sigma$ and $f \vdash \sigma \longrightarrow \sigma^{\prime}$, then $f, P_{\text {sem }} \vdash \sigma^{\prime}$.

As we show next, Theorem 8 can be used to justify the correctness of many SSA-based transformations. Instantiating $P$ with other predicates can also be useful—Section 5 shows how.

\subsection{The correctness of SSA-based transformations}

Consider again the example code transformation from Figure 2. It, and many other SSA-based optimizations, can be defined by using a combination of simpler transformations: deleting an unused definition, substituting a constant expression for a variable, substituting one variable by another, or moving variable definitions. Each such transformation is subject to the SSA constraints-for example, we can't move a definition later than one of its uses - and each transformation preserves the SSA invariants. By pipelining these basic transformations, we can define more sophisticated SSA-based program transformations whose correctness is established by the composition of the proofs for the basic transformations.

In general, an SSA-based transformation from $f$ to $f^{\prime}$ is correct if it preserves both well-formedness and program behavior.

1. Preserving well-formedness: if $\vdash f$, then $\vdash f^{\prime}$.

2. Program refinement: if $\vdash f$, then $f \supseteq f^{\prime}$ (see Section 2.1).

Each of the basic transformations mentioned above can be proved correct by using Theorem 8 . For the sake of space, here we present only the correctness of variable substitution (though we proved correct all the mentioned transformations in our Coq development). Section 4 shows how to extend the transformations to implement memory-aware optimizations in the full Vellvm.

Variable substitution Consider the step of the program transformation from Figure 2 in which the use of $r_{8}$ on the last line is replaced by $r_{4}$ (this is valid only after hoisting the definition of $r_{4}$ so that it is in scope). This transformation is correct because both $r_{4}$ and $r_{8}$ denote the same value, and the definition of $r_{4}$ (after hoisting) strictly dominates the definition of $r_{8}$. In Figure 2, it is enough to do redundant variable elimination - this optimization lets us replace one variable by another when their definitions are syntactically equal; other optimizations, such as global value numbering, allow a coarser, more semantic, equality to be used. Proving them correct follows the same basic pattern as the proof shown below.

DEFINITION 3 (Redundant Variable). In a function $f$, a variable $r_{2}$ is redundant with variable $r_{1}$ if:

1. $f$ defines $r_{1} @ p c_{1}, f$ defines $r_{2} @ p c_{2}$ and $f \vdash p c_{1} \succ p c_{2}$

2. $f\left[p c_{1}\right]=\left\lfloor c_{1}\right\rfloor, f\left[p c_{1}\right]=\left\lfloor c_{2}\right\rfloor$ and $c_{1}$ and $c_{2}$ have syntactically equal right-hand-sides.

We would like to prove that eliminating a redundant variable is correct, and therefore must relate a program $f$ with $f\left\{r_{1} / r_{2}\right\}$. Since substitution does not change the control-flow graph, it preserves the domination relations.

\section{LEMMA 9}

1. $f \vdash l_{1} \succcurlyeq l_{2} \Longleftrightarrow f\left\{r_{2} / r_{1}\right\} \vdash l_{1} \succcurlyeq l_{2}$

2. $f \vdash p c_{1} \succ p c_{2} \Longleftrightarrow f\left\{r_{2} / r_{1}\right\} \vdash p c_{1} \succ p c_{2}$

Applying Lemma 1 and Lemma 9, we have:

Lemma 10. Suppose that in $f, r_{1}$ is redundant with $r_{2}$. If $\vdash f$, then $\vdash f\left\{r_{2} / r_{1}\right\}$.

Let two program states simulate each other if they have the same local state $\delta$ and program counter. We assume that the original program and its transformation have the same initial state.

LEMMA 11. If $\vdash f, r_{2}$ is redundant with $r_{1}$ in $f$, and $(p c, \delta)$ is a reachable state, then

1. If val is an operand of a non-phinode at program counter $p c$, then $\exists v$. $\llbracket v a l \rrbracket_{\delta}=\lfloor v\rfloor \wedge \llbracket v a l\left\{r_{1} / r_{2}\right\} \rrbracket_{\delta}=\lfloor v\rfloor$.

2. If pc is $l_{i} . \mathbf{t}$, and $l_{i}$ is a previous block of a block with $\phi$-nodes ${\overline{\phi_{j}}}^{j}$, then $\exists \delta^{\prime} . \llbracket{\overline{\phi_{j}}}^{j} \rrbracket_{\delta}^{l_{i}}=\left\lfloor\delta^{\prime}\right\rfloor \wedge \llbracket{\overline{\phi_{j}}\left\{r_{1} / r_{2}\right\}}^{j} \rrbracket_{\delta}^{l_{i}}=\left\lfloor\delta^{\prime}\right\rfloor$.

Proof (sketch): The proof makes crucial use of Theorem 8. For example, to show part 1 for a source instruction $r:=r h s$ (with transformed instruction $r:=r h s\left\{r_{1} / r_{2}\right\}$ ) located at program counter $p c$, we reason like this: if $r_{2}$ is an operand used by $r h s$, then $r_{2} \in \operatorname{sdom}_{f}(p c)$ and by Theorem 8, property $P_{\text {sem }}$, implies that $\delta\left[r_{2}\right]=\llbracket r h s_{2} \rrbracket_{\delta}$ for some $r h s_{2}$ defining $r_{2}$. Since $r_{1}$ is used as an operand in $r h s\left\{r_{1} / r_{2}\right\}$, similar reasoning shows that $\delta\left[r_{1}\right]=$ 


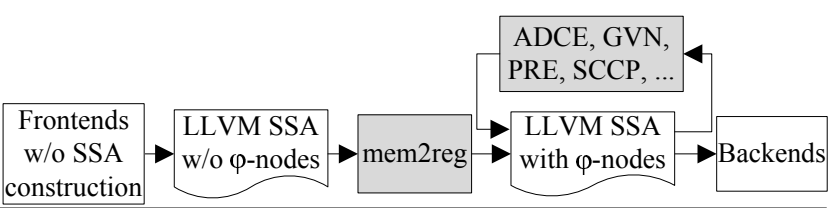

Figure 5. The tool chain of the LLVM compiler

$\llbracket r h s_{1} \rrbracket_{\delta}$, but since $r_{2}$ is redundant with $r_{1}$, we have $r h s_{2}=r h s_{1}$, and the result follows immediately.

Using Lemma 11, we can easily show the lock-step simulation lemma, which completes the correctness proof:

LEMMA 12. If $\vdash f, r_{2}$ is redundant with $r_{1}$ in $f, f\left\{r_{1} / r_{2}\right\} \vdash$ $\sigma_{1} \longrightarrow \sigma_{2}$, then $f \vdash \sigma_{1} \longrightarrow \sigma_{2}$.

\section{LLVM and mem2reg}

From Vminus to Vellvm Vminus provides a convenient minimal setting in which to study SSA-based optimizations, but it omits many features necessary in a real intermediate representation. To demonstrate that our proof techniques can be used for practical compiler optimizations, we next show how to apply them to the LLVM IR used in Vellvm [22].

LLVM [10] (Low-Level Virtual Machine) is a robust, industrialstrength, and open-source compilation framework that competes with GCC in terms of compilation speed and performance of the generated code. LLVM uses a platform-independent SSA-based intermediate representation [10], and provides a large suite of optimization passes, including aggressive dead code elimination, global value numbering, partial redundancy elimination, and sparse conditional constant propagation, among others. Each transformation pass consumes and produces code in this SSA form, and they typically have the flavor of the code transformations described above in Section 3. Figure 5 depicts LLVM's tool chain.

The Vellvm infrastructure provides a Coq implementation of the full LLVM 3.0 intermediate language and defines (several) operational semantics along with some useful metatheory about the memory model. Figure 6 shows the additional Vellvm features needed to explain the following proofs; more details about the operational semantics can be found in our earlier Vellvm work [22].

A program prog includes a list of products prod that are either global constants or function definitions. The address of a global constant with type typ is a constant typ $*$ gid of type typ $*$. Vellvm also has memory operations that include stack allocation, loads, and stores. At runtime, a pointer in is represented by a block name blk and an offset ofs within the block. All globals are allocated before the start of a program and stored in a mapping $g$. A program state $S$ is composed of a memory state $M$ and a list of stack states $\bar{\sigma}$. We denote small-step evaluation by config $\vdash S \stackrel{t}{\longrightarrow} S^{\prime}$ where a program configuration config includes a program prog and globals. Judgment $\vdash$ prog denotes a well-formed program-it checks that all definitions of globals and functions are unique, each function is well-formed, etc.

\subsection{The mem2reg Optimization Pass}

A critical piece of LLVM's compilation strategy is the mem2reg pass, which takes code that is "trivially" in SSA form and converts it into a minimal, pruned SSA program [15]. This strategy simplifies LLVM's many front ends by moving work in to mem 2 reg. An SSA form is "minimal" if each $\phi$ is placed only at the dominance frontier of the definitions of the $\phi$ node's incoming variables [7]. A minimal SSA form is "pruned" if it contains only live $\phi$ nodes [15]. This pass enables many subsequent optimizations (and, in particular, backend optimizations such as register allocation) to work effectively.

\begin{tabular}{|c|c|c|c|}
\hline Programs & prog & $::=$ & $\overline{\text { prod }}$ \\
\hline Products & prod & $::=$ & gid $=$ global typ const $\mid f$ \\
\hline Functions & $f$ & $::=$ & define typ fid $(\overline{a r g})\{\bar{b}\}$ \\
\hline Types & typ & $::=$ & .. $\mid$ typ $*$ \\
\hline Constants & cnst & $::=$ & $\cdots \quad$ typ $*$ gid \\
\hline Commands & $c$ & $:=$ & $\begin{array}{l}\cdots \\
r:=\text { alloca typ } \\
r:=\text { load }(\text { typ } *) r_{1} \\
\text { store typ } \text { val }_{1} r_{2} \\
\text { option } r=\text { call typ fid } \overline{\text { param }}\end{array}$ \\
\hline Values & $v$ & $::=$ & $\cdots \mid$ blk.ofs \\
\hline Globals & $g$ & $::=$ & $r \mapsto v$ \\
\hline Configurations & config & $::=$ & prog, $g$ \\
\hline Allocas & $\alpha$ & $::=$ & $\emptyset \mid b l k, \alpha$ \\
\hline Frames & $\sigma$ & $::=$ & $(f, p c, \delta, \alpha)$ \\
\hline Call stacks & $\bar{\sigma}$ & $::=$ & $\emptyset \mid \sigma, \bar{\sigma}$ \\
\hline Program states & $S$ & $::=$ & $M, \bar{\sigma}$ \\
\hline
\end{tabular}

Figure 6. The syntax and program states of Vellvm (excerpt)

Figure 7 demonstrates the importance of the mem2reg pass for LLVM's generated code performance. In our experiments, running only the mem 2 reg pass yields a $81 \%$ speedup (on average) compared to LLVM without any optimizations; doing the full suite of -01 level optimizations (which includes mem2reg) yields a speedup of $102 \%$, which means that mem 2 reg alone captures all but $\% 12$ of the benefit of the -01 level optimizations. Comparison with -03 optimizations yields similar results. These observations make mem 2 reg an obvious target for our verification efforts.

The "trivial" SSA form is generated directly by compiler front ends, and it uses the alloca instruction to allocate stack space for every source-program local variable and temporary needed. In this form, an LLVM SSA variable is used either only locally to access those stack slots, in which case the variable is never live across two basic blocks, or it is a reference to the stack slot, whose lifetime corresponds to the source-level variable's scope. These constraints mean that no $\phi$ instructions are needed-it is extremely straightforward for a front end to generate code in this form.

As an example, consider this $\mathrm{C}$ program:

$$
\text { int } i=0 \text {; while }(i<=100) \quad i++ \text {; return } i \text {; }
$$

The "trivial" SSA form that might be produced by the frontend of a compiler is shown in the left-most column of Figure 8. The $r_{0}:=$ alloca int instruction on the first line allocates space for the source variable $i$, and $r_{0}$ is a reference from which local load and store instructions access $i$ 's contents.

The mem2 reg pass converts promotable uses of stack-allocated variables to SSA temporaries. An alloca'ed variable like $r_{0}$ is considered to be promotable, written promotable $\left(f, r_{0}\right)$, if it is created in the entry block of function $f$ and it doesn't escapei.e., its value is never written to memory or passed as an argument to a function call. The mem 2 reg pass identifies promotable stack allocations and then replaces them by temporary variables in SSA form. It does this by placing $\phi$ nodes, substituting each variable defined by a load with the previous value stored into the stack slot, and then eliminating the memory operations (which are now dead). The right-most column of Figure 8 shows the resulting pruned SSA program for this example.

Proving that mem 2 reg is correct is nontrivial because it makes significant, non-local changes to the use of memory locations and temporary variables. Furthermore, the specific mem2reg algorithm used by LLVM is not directly amenable to the proof techniques developed in Section 3-it was not designed with verification in mind, so it produces intermediate stages that break the SSA invariants or do not preserve semantics. This section therefore describes an alternate algorithm that is more suitable to formalization. 


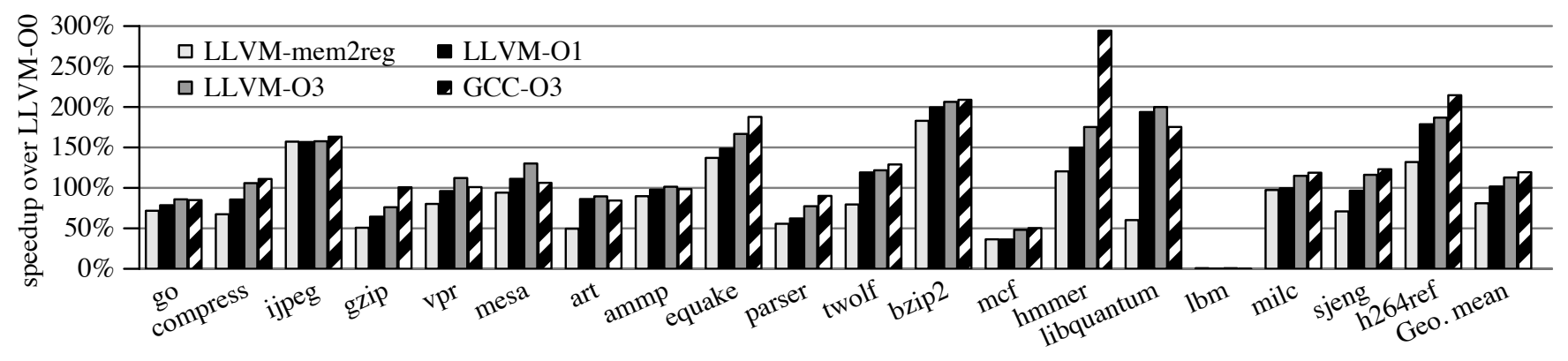

Figure 7. Normalized execution time improvement of the LLVM's mem2reg, LLVM's O1, and LLVM's O3 optimizations over the LLVM baseline with optimizations disabled. For comparison, GCC-O3's speedup over the same baseline is also shown.

\begin{tabular}{|c|c|c|c|c|}
\hline "trivial" SSA & Maximal $\phi$ nodes placement & After LAS/LAA/SAS & After DSE/DAE & After $\phi$ nodes elimination \\
\hline $\begin{aligned} l_{1}: r_{0}:=\text { alloca int } \\
\text { store int } 0 r_{0}\end{aligned}$ & $\begin{array}{l}l_{1}: r_{0}:=\text { alloca int } \\
\quad \text { store int } 0 r_{0} \\
\quad r_{7}:=\text { load }(\text { int } *) r_{0}\end{array}$ & $\begin{aligned} l_{1}: r_{0}:=\text { alloca int } & \text { store int } 0 r_{0}\end{aligned}$ & $l_{1}:$ & $l_{1}:$ \\
\hline$l_{2}:$ br $l_{2}$ & $\begin{array}{c}\mathbf{b r} l_{2} \\
l_{2}: r_{6}=\text { phi }\left[r_{7}, l_{1}\right]\left[r_{9}, l_{3}\right]\end{array}$ & $\begin{array}{c}\mathbf{b r} l_{2} \\
l_{2}: r_{6}=\text { phi }\left[0, l_{1}\right]\left[r_{9}, l_{3}\right]\end{array}$ & $\begin{array}{c}\mathbf{b r} l_{2} \\
l_{2}: r_{6}=\text { phi }\left[0, l_{1}\right]\left[r_{4}, l_{3}\right]\end{array}$ & $\begin{array}{c}\mathbf{b r} l_{2} \\
l_{2}: r_{6}=\text { phi }\left[0, l_{1}\right]\left[r_{4}, l_{3}\right]\end{array}$ \\
\hline$r_{1}:=\operatorname{load}(\operatorname{int} *) r_{0}$ & $\begin{array}{l}\text { store int } r_{6} r_{0} \\
r_{1}:=\operatorname{load}(\text { int } *) r_{0}\end{array}$ & store int $r_{6} r_{0}$ & & \\
\hline$r_{2}:=r_{1} \leq 100$ & $\begin{array}{l}r_{2}:=r_{1} \leq 100 \\
r_{8}:=\operatorname{load}(\text { int } *) r_{0}\end{array}$ & $r_{2}:=r_{6} \leq 100$ & $r_{2}:=r_{6} \leq 100$ & $r_{2}:=r_{6} \leq 100$ \\
\hline$l_{3}: \quad$ br $r_{2} l_{3} l_{4}$ & $\begin{array}{l}\mathbf{b r} r_{2} l_{3} l_{4} \\
l_{3}: r_{10}=\text { phi }\left[r_{8}, l_{2}\right] \\
\quad \text { store int } r_{10} r_{0}\end{array}$ & $\begin{array}{l}\mathbf{b r} r_{2} l_{3} l_{4} \\
l_{3}: r_{10}=\mathbf{p h i}\left[r_{6}, l_{2}\right]\end{array}$ & $\begin{array}{c}\mathbf{b r} r_{2} l_{3} l_{4} \\
l_{3}: r_{10}=\text { phi }\left[r_{6}, l_{2}\right]\end{array}$ & $l_{3}: \quad \mathbf{b r} r_{2} l_{3} l_{4}$ \\
\hline$r_{3}:=\operatorname{load}(\operatorname{int} *) r_{0}$ & $r_{3}:=\operatorname{load}($ int $*) r_{0}$ & & & \\
\hline $\begin{array}{l}r_{4}:=r_{3}+1 \\
\text { store int } r_{4} r_{0}\end{array}$ & $\begin{array}{l}r_{4}:=r_{3}+1 \\
\text { store int } r_{4} r_{0} \\
r_{9}:=\operatorname{load}(\text { int } *) r_{0}\end{array}$ & $\begin{array}{l}r_{4}:=r_{10}+1 \\
\text { store int } r_{4} r_{0}\end{array}$ & $r_{4}:=r_{10}+1$ & $r_{4}:=r_{6}+1$ \\
\hline br $l_{2}$ & br $l_{2}$ & br $l_{2}$ & br $l_{2}$ & br $l_{2}$ \\
\hline$l_{4}$ & $\begin{array}{c}l_{4}: r_{11}=\text { phi }\left[r_{8}, l_{2}\right] \\
\quad \text { store int } r_{11} r_{0}\end{array}$ & $\begin{aligned} l_{4}: & r_{11}=\text { phi }\left[r_{6}, l_{2}\right] \\
& \text { store int } r_{11} r_{0}\end{aligned}$ & $l_{4}: r_{11}=$ phi $\left[r_{6}, l_{2}\right]$ & $l_{4}:$ \\
\hline $\begin{array}{l}r_{5}:=\operatorname{load}(\text { int } *) r_{0} \\
\text { ret int } r_{5}\end{array}$ & $\begin{array}{l}r_{5}:=\operatorname{load}(\text { int } *) r_{0} \\
\text { ret int } r_{5}\end{array}$ & ret int $r_{11}$ & ret int $r_{11}$ & ret int $r_{6}$ \\
\hline
\end{tabular}

Figure 8. The SSA construction by the vmem2reg pass

\subsection{The vmem2 reg Algorithm}

The vmem2reg algorithm is structured to lead to a clean formalism and yet still produce programs with effectiveness similar to the LLVM mem2reg pass. To demostrate the main ideas of vmem2reg, this section describes an algorithm that uses straightforward micro-pass pipelining. Section 6 presents a smarter way to "fuse" the micro passes, thereby reducing compilation time. Proving pipeline fusion correct is (by design) independent of the proofs for the vmem2reg algorithm shown in the section.

At a high level, vmem2reg (whose code is shown in Figure 10) traverses all functions of the program, applying the transformation vmem 2 reg_f $n$ to each. Figure 9 depicts the main loop, which is an extension of Aycock and Horspool's SSA construction algorithm [3]. vmem2reg_fn first iteratively promotes each promotable alloca by adding $\phi$ nodes at the beginning of every block. After processing all promotable allocas, vmem2reg_fn removes redundant $\phi$ nodes, and eventually will produce a program almost in pruned SSA form, ${ }^{3}$ in a manner similar to previous algorithms [15].

\footnotetext{
${ }^{3}$ Technically, fully pruned SSA requires a more aggressive dead- $\phi$ elimination pass that we omit for the sake of simplicity. Section 7 shows that this omission is negligible.
}

The transformation that vmem2reg_fn applies to each function is a composition of a series of micro transformations (LAS, LAA, SAS, DSE, and DAE, shown in Figure 9). Each of these transformations preserves the well-formedness and semantics of its input program; moreover, these transformations are relatively small and local, and can therefore be reasoned about more easily.

At each iteration of alloca promotion, vmem2reg_fn finds a promotable allocation $r$. Then $\phi$-nodes_placement (code shown in Figure 10) adds $\phi$ nodes for $r$ at the beginning of every block. To preserve both well-formedness and the original program's semantics, $\phi$-nodes_placement also adds additional loads and stores around each inserted $\phi$ node. At the end of every block that has successors, $\phi$-nodes_placement introduces a load from $r$, and stores the result in a fresh temporary; at the beginning of every block that has a predecessor, $\phi$-nodes_placement first inserts a fresh $\phi$ node whose incoming value from a predecessor $l$ is the value of the corresponding load added at the end of $l$, then inserts a store to $r$ with the value of the new $\phi$ node.

The second column in Figure 8 shows the result of running the $\phi$-node placement pass starting from the example program in its trivial SSA form. It is not difficult to check that this code is in SSA form. Moreover, the output program also preserves the meaning of the original program. For example, at the end of block $l_{1}$, the pro- 


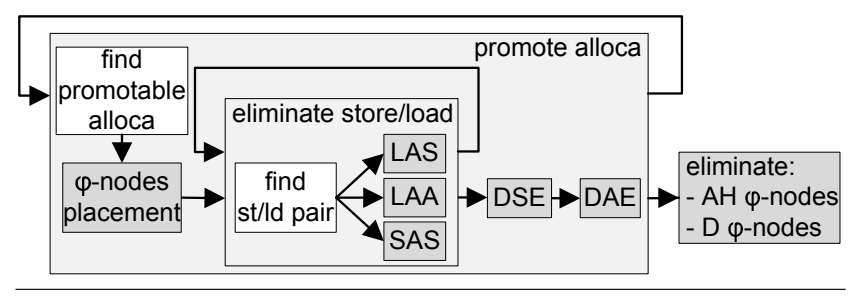

Figure 9. Basic structure of vmem 2 reg_fn

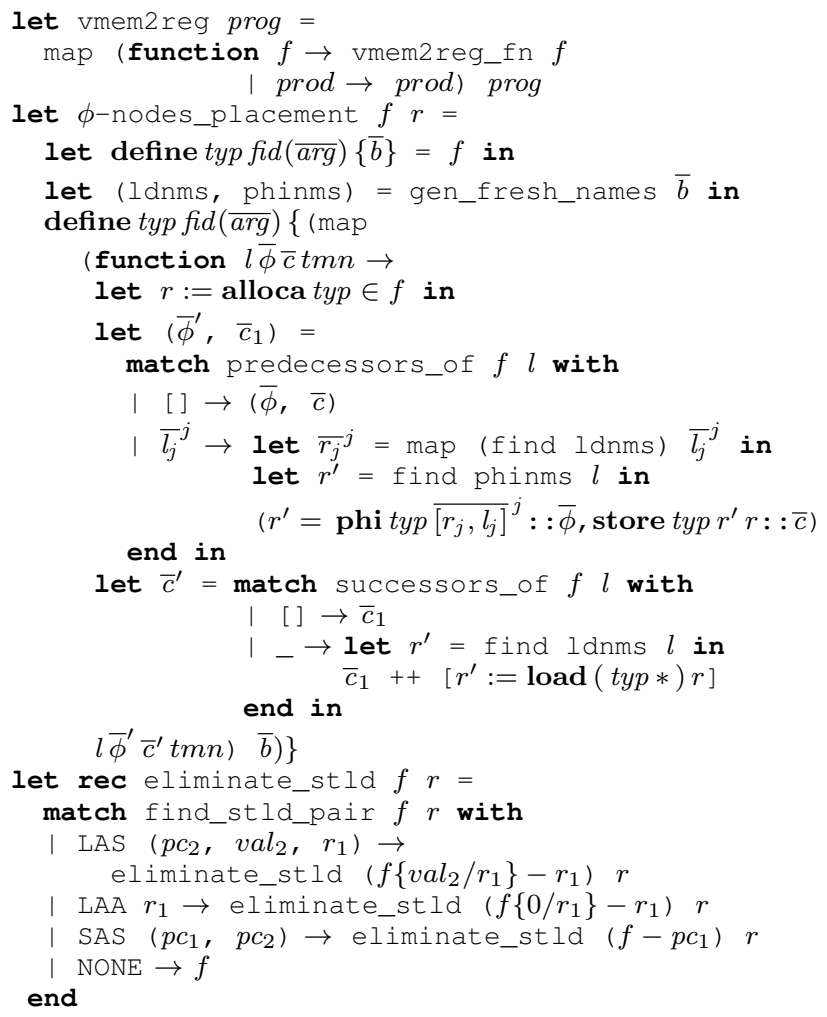

Figure 10. The algorithm of vmem2reg

gram loads the value stored at $r_{0}$ into $r_{7}$. After jumping to block $l_{2}$, the value of $r_{7}$ is stored into the location $r_{0}$, which should contain the same values as $r_{7}$. Therefore, the additional store does not change the status of memory. Although the output program contains more temporaries than the original program, these temporaries are used only to connect inserted loads and stores, and so they do not interfere with the original temporaries.

To remove the additional loads and stores introduced by the $\phi$-node placement pass and eventually promote allocas to registers, vmem2reg_fn next applies a series of micro program transformations until no more optimizations can be applied.

First, vmem 2 reg_f $n$ iteratively does the following transformations (implemented by eliminate_st ld shown in Figure 10):

1. LAS $\left(r_{1}, p c_{2}, v a l_{2}\right)$ "Load After Store": $r_{1}$ is loaded from $r$ after a store of $v a l_{2}$ to $r$ at program counter $p c_{2}$, and there are no other stores of $r$ in any path (on the control-flow graph) from $p c_{2}$ to $r_{1}$. In this case, all uses of $r_{2}$ can be replaced by $v l_{2}$, and the load can be removed.

2. LAA $r_{1}$ "Load After Alloca": As above, but the load is from an uninitialized memory location at $r . r_{1}$ can be replaced by LLVM's default memory value, and the load can be removed.
3. SAS $\left(p c_{1}, p c_{2}\right)$ : The store at program counter $p c_{2}$ is a store after the store at program counter $p c_{1}$. If both of them access $r$, and there is no load of $r$ in any path (on the control-flow graph) from $p c_{1}$ to $p c_{2}$, then the store at $p c_{1}$ can be removed.

At each iteration step of eliminate_stld, the algorithm uses the function $f i n d \_s t l d \_p a i r$ to identify each of the above cases. Because the $\phi$-node placement pass only adds a store and a load as the first and the last commands at each block respectively, find_st ld_pair only needs to search for the above cases within blocks. This simplifies both the implementation and proofs. Moreover, eliminate_stld must terminate because each of its transformations removes one command. The third column in Figure 8 shows the code after eliminate_stld.

Next, the algorithm uses DSE (Dead Store Elimination) and DAE (Dead Alloca Elimination) to remove the remaining unnecessary stores and allocas. The fourth column in Figure 8 shows the code after DSE and DAE.

Finally, vmem2reg_fn eliminates unnecessary and dead $\phi$ nodes. A $\phi$-node is unnecessary [3] if $\phi$ is of the form $r=$ phi typ ${\overline{\left[v a l_{j}, l_{j}\right]}}^{j}$ where all the $v a l_{j}$ 's are either equal to $r$ or to val. In this case, uses of $r$ can be replaced by val, and the $\phi$ node can be removed. Aycock and Horspool [3] proved that when there is no such $\phi$ node in a reducible program, the program is of the minimal SSA form. The right-most column in Figure 8 shows the final output of the algorithm.

\section{Correctness of vmem 2 reg}

We prove the correctness of vmem2reg using the techniques developed in Section 3. At a high level, the correctness of vmem 2 reg is the composition of the correctness of each micro transformation of vmem 2 reg shown in Figure 10. Given a well-formed input program, each shaded box must produce a well-formed program that preserves the semantics of the input program. Moreover, the micro transformations except $\mathrm{DAE}$ and $\phi$-nodes elimination must preserve the promotable predicate, because the correctness of subsequent transformations relies on fact that promotable allocations aren't aliased.

Formally, let $\operatorname{prog}\left\{f^{\prime} / f\right\}$ be the substitution of $f$ by $f^{\prime}$ in $\operatorname{prog}$, and let $(f)$ be a micro transformation of $f$ applied by vmem 2 reg. (-) must satisfy:

1. Preserving promotable: when (-D) is not DAE or $\phi$-nodes elimination, if promotable $(f, r)$, then promotable $(0 f), r)$.

2. Preserving well-formedness: if promotable $(f, r)$ when (1-D is $\phi$-nodes placement, and $\vdash \operatorname{prog}$, then $\vdash \operatorname{prog}\{(f) / f\}$.

3. Program refinement: if promotable $(f, r)$ when $\left(D_{-}\right)$is not $\phi$ nodes elimination, and $\vdash \operatorname{prog}$, then $\operatorname{prog} \supseteq \operatorname{prog}\{(f) / f\}$.

\subsection{Preserving promotability}

At the beginning of each iteration for promoting allocas, the algorithm indeed finds promotable allocations.

LEMMA 13. If prog $\vdash f$, and vmem2reg_fn finds a promotable allocation $r$ in $f$, then promotable $(f, r)$.

We next show that $\phi$-nodes placement preserves promotable:

LEMMA 14. If promotable $(f, r)$,

then promotable ( $\phi$-nodes_placement $f r, r)$.

Each of the other micro transformations is composed of one or two more basic transformations: variable substitution, denoted by $f\{v a l / r\}$, and instruction removal, denoted by filter check $f$ where filter removes an instruction insn from $f$ if check insn $=$ 


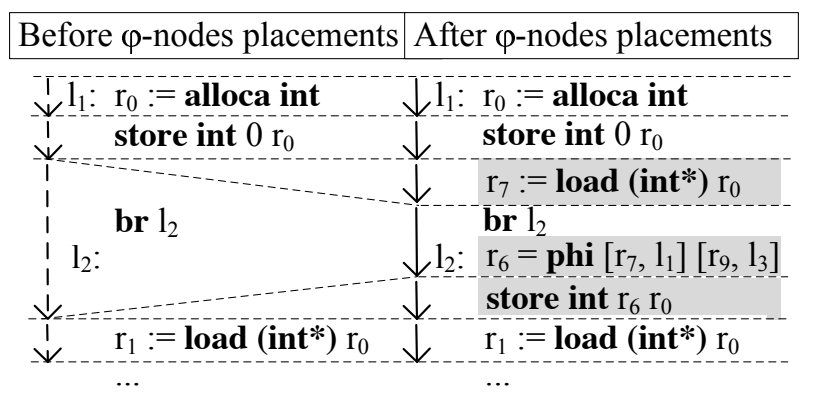

Figure 11. The simulation relation for $\phi$-node placement

false. For example, $f\left\{v l_{2} / r_{1}\right\}-r_{1}$ (LAS) is a substitution followed by a removal in which check insn = false iff insn defines $r_{1}$; DSE of a promotable alloca $r$ is a removal in which check $i n s n=$ false iff insn is a store to $r$. We first establish that substitution and removal preserve promotable.

LEMMA 15. Suppose promotable $(f, r)$,

1. If $\neg\left(v_{a l}\right.$ uses $\left.r\right)$, then promotable $\left(f\left\{v a l_{1} / r_{1}\right\}, r\right)$.

2. If check insn $=$ false $\Rightarrow$ insn does not define $r$, then promotable (filter check $f, r)$.

We can show that the other micro transformations preserve promotable by checking the preconditions of Lemma 15.

LEMMA 16. Suppose promotable $(f, r), r$ is still promotable after LAS, LAA, SAS or DSE.

The substituted value of LAS is written to memory by a store in $f$, which cannot use $r$ because $r$ is promotable in $f$. The substituted value of LAA is a constant that cannot use $r$ trivially. Moreover, LAS, LAA, SAS and DSE remove only loads or stores.

\subsection{Preserving well-formedness}

It is sufficient to check the following conditions to show that a function-level transformation preserves well-formedness:

\section{LEMMA 17. Suppose}

1. $(f)$ and $f$ have the same signature.

2. if prog $\vdash f$, then $\operatorname{prog}\{(f) / f\} \vdash(f)$.

If $\vdash \operatorname{prog}$, then $\vdash \operatorname{prog}\{(f) / f\}$.

It is easy to see that all transformations vmem2reg applies satisfy the first condition. We first prove that $\phi$-nodes placement preserves the second condition:

LEMMA 18. If promotable $(f, r)$, prog $\vdash f$ and let $f^{\prime}$ be $\phi$-nodes_placement $f r$, then prog $\left\{f^{\prime} / f\right\} \vdash f^{\prime}$.

Similarly, to reason about other transformations, we first establish that substitution and removal preserve well-formedness.

LEMMA 19. Suppose prog $\vdash f$,

1. If $f \vdash v a l_{1} \succ r_{2}, f^{\prime}=f\left\{v a l_{1} / r_{2}\right\}$, then $\operatorname{prog}\left\{f^{\prime} / f\right\} \vdash f^{\prime}$.

2. If check insn $=$ false $\Rightarrow f$ does not use insn, and let $f^{\prime}$ be filter check $f$, then $\operatorname{prog}\left\{f^{\prime} / f\right\} \vdash f^{\prime}$.

Here, $f \vdash v a l_{1} \succ r_{2}$ if $f \vdash r_{1} \succ r_{2}$ when $v l_{1}$ uses $r_{1}$. Note that the first part of Lemma 19 is an extension of Lemma 10 that only allows substitution on commands. In vmem2reg, LAS and $\phi$-nodes elimination may transform $\phi$-nodes.

LAS, LAA and $\phi$-nodes elimination remove instructions after substitution. The following auxiliary lemma shows that the substituted definition is removable after substitution (by Lemma 2):

LEMMA 20. If $f \vdash v a l_{1} \succ r_{2}$, then $f\left\{v a l_{1} / r_{2}\right\}$ does not use $r_{2}$.
By Lemma 19, Lemma 20 and the proofs [3], we have:

LEMMA 21. LAS, LAA, SAS, DSE, DAE and $\phi$-nodes elimination preserve well-formedness.

\subsection{Program refinement}

The proofs of program refinement use the simulation diagrams in Section 2 and different instantiations of the GWF_FR rule we developed in Section 3, where instead of just a function $f$ and frame $\sigma$, we now have a configuration config that also includes the program memory.

Promotability As we discussed above, the micro transformations (except $\phi$-nodes elimination) rely on the promotable property. We start by establishing the invariants related to promotability, namely that promotable allocations aren't aliased. This proof is itself an application of GWF_FR.

The promotable property ensures that a promotable alloca of a function does not escape-the function can access the data stored at the allocation, but cannot pass the address of the allocation to other contexts. Therefore, in the program, the promotable alloca and all other pointers (in memory, local temporaries and temporaries on the stack) must not alias. Formally, given a promotable allocation $r$ with type typ* in $f$, we define $P_{\text {noalias }}(f, r$, typ $)$ :

$$
\begin{aligned}
& \lambda \text { config. } \lambda S \text {. } \\
& \forall \bar{\sigma}_{1}++\sigma:: \bar{\sigma}_{2}=S . \bar{\sigma} . f=\sigma . f \wedge \llbracket r \rrbracket_{\sigma . \delta}=\lfloor b l k\rfloor \Longrightarrow \\
& \exists v \cdot \operatorname{load}(S . M, \text { typ }, b l k)=\lfloor v\rfloor \\
& \wedge \quad \forall b l k^{\prime} . \forall t y p^{\prime} . \neg \operatorname{load}\left(S . M, t y p^{\prime}, b l k^{\prime}\right)=\lfloor b l k\rfloor \\
& \wedge \forall r^{\prime} \neq r \Longrightarrow \neg \llbracket r^{\prime} \rrbracket_{\sigma . \delta}=\lfloor b l k\rfloor \\
& \wedge \forall \sigma^{\prime} \in \bar{\sigma}_{1} . \forall r^{\prime} . \neg \llbracket r^{\prime} \rrbracket \sigma^{\prime} . \delta=\lfloor b l k\rfloor
\end{aligned}
$$

The last clause ensures that the alloca and the variables in the callees reachable from $f$ do no alias. In CompCert, the translation from C\#minor to Cminor uses properties (in non-SSA form) similar to $P_{\text {noalias }}(f, r$, typ $)$ to allocate local variables on stack.

LEMMA 22 (A promotable alloca is not aliased). At any reachable program state $S$, config, $P_{\text {noalias }}(f, r$, typ $) \vdash S$ holds.

The invariant holds initially. At all reachable states, the invariant holds because a promotable allocation cannot be copied to other temporaries, stored to memory, passed into a function, or returned. Therefore, in a well-defined program no external code can get its location by accessing other temporaries and memory locations. Importantly, the memory model ensures that from a consistent initial memory state, all memory blocks in temporaries and memory are allocated-it is impossible to forge a fresh pointer from an integer.

$\phi$-node placement Figure 11 pictorially shows an example (which is the code fragment from Figure 8 ) of the simulation relation $\sim$ for proving that the $\phi$-node placement preserves semantics. It follows left "option" simulation, because $\phi$-node placement only inserts instructions. We use the number of unexecuted instructions in the current block as the measure function.

The dashed lines indicate where the two program counters must be synchronized. Although the pass defines new variables and stores (shaded in Figure 11), the variables are only passed to the new $\phi$ nodes, or stored into the promotable allocation; additional stores only update the promotable allocation with the same value. Therefore, by Lemma 22, requires that two programs have the same memory states and the original temporaries match.

LEMMA 23.

If $f^{\prime}=\phi$-nodes_placement $f r$, and promotable $(f, r)$, and $\vdash$ prog, then prog $\supseteq \operatorname{prog}\left\{f^{\prime} / f\right\}$. 


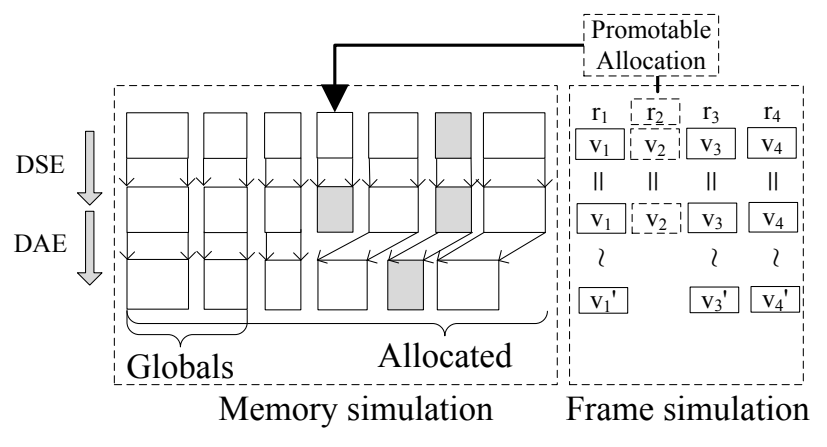

Figure 12. The simulation relation for $D S E$ and $D A E$

The interesting case is to show that $\sim$ implies a correspondence between stuck states. Lemma 22 ensures that the promotable allocation cannot be dereferenced by operations on other pointers. Therefore, the inserted memory accesses are always safe.

LAS/LAA We present the proofs for the correctness of LAS. The proofs for the correctness of LAA is similar. In the code after $\phi$ node placement of Figure $8, r_{7}:=\operatorname{load}($ int $*) r_{0}$ is an LAS of store int $0 r_{0}$. We observe that at any program counter $p c$ between the store and load, the value stored at $r_{0}$ must be 0 because alive $\left(p c_{1}, p c_{2}\right)$ holds- the store defined at $p c_{1}$ is not overwritten by other writes until $p c$.

To formalize the observation, consider a promotable $r$ with type typ $*$ in $f$. Suppose $\mathrm{find}$ _st $1 \mathrm{~d}_{-}$pair $f r=$ LAS $\left(p c_{2}\right.$, $\left.v_{a l}, r_{1}\right)$. Consider the invariant $P_{\text {las }}\left(f, r, \operatorname{typ}, p c_{2}, v a l_{2}\right)$ :

$$
\begin{aligned}
& \lambda c o n f i g . \lambda S . \forall \sigma \in S . \bar{\sigma} . \\
& \quad\left(f=\sigma . f \wedge \llbracket v a l_{2} \rrbracket_{\sigma . \delta}=\left\lfloor v_{2}\right\rfloor \wedge \llbracket r \rrbracket_{\sigma . \delta}=\lfloor b l k\rfloor \wedge\right. \\
& \left.\quad \text { alive }\left(p c_{2}, \sigma . p c\right)\right) \Longrightarrow \operatorname{load}(S . M, \text { typ }, b l k)=\left\lfloor v_{2}\right\rfloor
\end{aligned}
$$

Using Lemma 22, we have that:

LEMMA 24. If promotable $(f, r)$, then alive $\left(p c_{2}, r_{1}\right)$ and at any reachable state $S$, config, $P_{\text {las }}\left(f, r\right.$, typ $\left., p c_{2}, v a l_{2}\right) \vdash S$ holds.

Let two programs relate to each other if they have the same program states. Lemma 24 establishes that the substitution in LAS is correct. The following lemma shows that removal of unused instructions preserves semantics in general.

LEMMA 25. If check insn $=$ false $\Rightarrow f$ does not use insn, and $\vdash$ prog, then prog $\supseteq \operatorname{prog}\{$ filter check $f / f\}$.

Lemma 20 shows that the precondition of Lemma 25 holds after the substitution in LAS. Finally, we have that:

LEMMA 26. LAS preserves semantics.

$S A S / D S E / D A E$ Here we discuss only the simulation relations used by the proofs. SAS removes a store to a promotable allocation overwritten by a following memory write. We consider a memory simulation that is the identity when the program counter is outside the SAS pair, but ignores the promotable alloca when the program counter is between the pair. Due to Lemma 22 and the fact that there is no load between a SAS pair, no temporaries or other memory locations can observe the value stored at the promotable alloca between the pair.

Figure 12 pictorially shows the simulation relations between the program states before and after DSE or DAE. Shaded memory blocks contain uninitialized values. The program states on the top are before DSE, where $r_{2}$ is a temporary that holds the promotable stack allocation and is not used by any loads. After DSE, the memory values for the promotable allocation may not match the original program's corresponding block. However, values in temporaries and all other memory locations must be unchanged (by
Lemma 22). Note that unmatched memory states only occur after the promotable allocation; before the allocation, the two memory states should be the same.

The bottom part of Figure 12 illustrates the relations between programs before and after DAE. After DAE, the correspondence between memory blocks of the two programs is not bijective, due to the removal of the promotable alloca. However, there must exist a mapping $\sim$ from the output program's memory blocks to the original program's memory blocks. The simulation requires that all values stored in memory and temporaries (except the promotable allocation) are equal modulo the mapping $\sim$.

$\phi$-nodes elimination Consider $r=$ phi typ ${\overline{\left[v a l_{j}, l_{j}\right]}}^{j}$ (an $\mathrm{AH}$ $\phi$-node) where all the $v_{a l}$ 's are either equal to $r$ or some $v a l^{\prime}$. Lemma 21 showed that $f \vdash v a l^{\prime} \succ r$. Intuitively, at any $p c$ that both $v a l^{\prime}$ and $r$ strictly dominate, the values of $v a l^{\prime}$ and $r$ must be the same. Consider the invariant $P_{\mathrm{ah}}\left(f, r, v a l^{\prime}\right)$ :

$$
\begin{aligned}
& \lambda \text { config. } \lambda S . \forall \sigma \in S . \bar{\sigma} . \\
& \quad f=\sigma . f \wedge \llbracket r \rrbracket_{\sigma . \delta}=\left\lfloor v_{1}\right\rfloor \wedge \llbracket v a l^{\prime} \rrbracket_{\sigma . \delta}=\left\lfloor v_{2}\right\rfloor \Longrightarrow v_{1}=v_{2}
\end{aligned}
$$

LEMMA 27. config, $P_{\mathrm{ah}}\left(f, r, v_{a l}^{\prime}\right) \vdash S$ holds for any reachable program state $S$.

Lemma 27 establishes that the substitution in $\phi$-nodes elimination is correct by using the identity relation. Lemma 20 and Lemma 25 show that removing dead $\phi$-nodes is correct.

\subsection{The correctness of vmem 2 reg}

Our main result, fully verified in Coq, is the composition of the correctness proofs for all the micro program transformations:

THEOREM 28 (vmem2reg is correct). If $f^{\prime}=$ vmem2reg $f$ and $\vdash \operatorname{prog}$, then $\vdash \operatorname{prog}\left\{f^{\prime} / f\right\}$ and $\operatorname{prog} \supseteq \operatorname{prog}\left\{f^{\prime} / f\right\}$.

\section{Pipeline Fusion}

In vmem2reg (show in Figure 9), there are two loops of micro pass-pipelinings: eliminate_stld and the redundant $\phi$-node elimination. This section gives a smarter pipelining vmem 2 reg-O that "fuses" the micro passes, reducing compilation time. For the sake of space, we present the fused version of eliminate_st ld; the fused $\phi$-node elimination follows similarly (see our Coq development). The design and correctness proofs described here should be applicable to other optimizations besides vmem2 reg.

At a high level, Figure 13 gives eliminate_stld_o, the fused eliminate_st ld, which first takes a pass to find all initial eliminations, fuses them, and then takes another pass that eliminates load's and store's in terms of fused eliminations.

We use actions $a c$ to denote micro eliminations:

$$
\text { Actions } a c \quad::=\quad r \mapsto v a l \mid p c \mapsto \#
$$

Here, $r \mapsto v a l$ denotes LAS $(r, p c, v a l)$ or LAA $r$ with the default memory value $v a l$ and $p c \mapsto \#$ denotes SAS $\left(p c, p c^{\prime}\right)$. We use $A C$ to denote a list of actions, and $A C(f)$ to denote a single pass of $f$ that, for each $r \mapsto v a l$, replaces all uses of $r$ by $v a l$, then removes the definition of $r$ and, for each $p c \mapsto \#$, removes the store at $p c$.

To find all initial actions, eliminate_stld_o traverses the blocks of a function, uses find_stld_pairs_block to find actions for each block, and then concatenates them. At each block, find_stld_pairs_block traverses each command (by find_stld_pairs_cmd), and uses stld_state to keep track of the search state: ST_INIT is the initial state; ST_AL typ records the element type of the promotable allocation; ST_ST $p c$ val records the value stored by the latest store at $p c$ to the promotable allocation. 


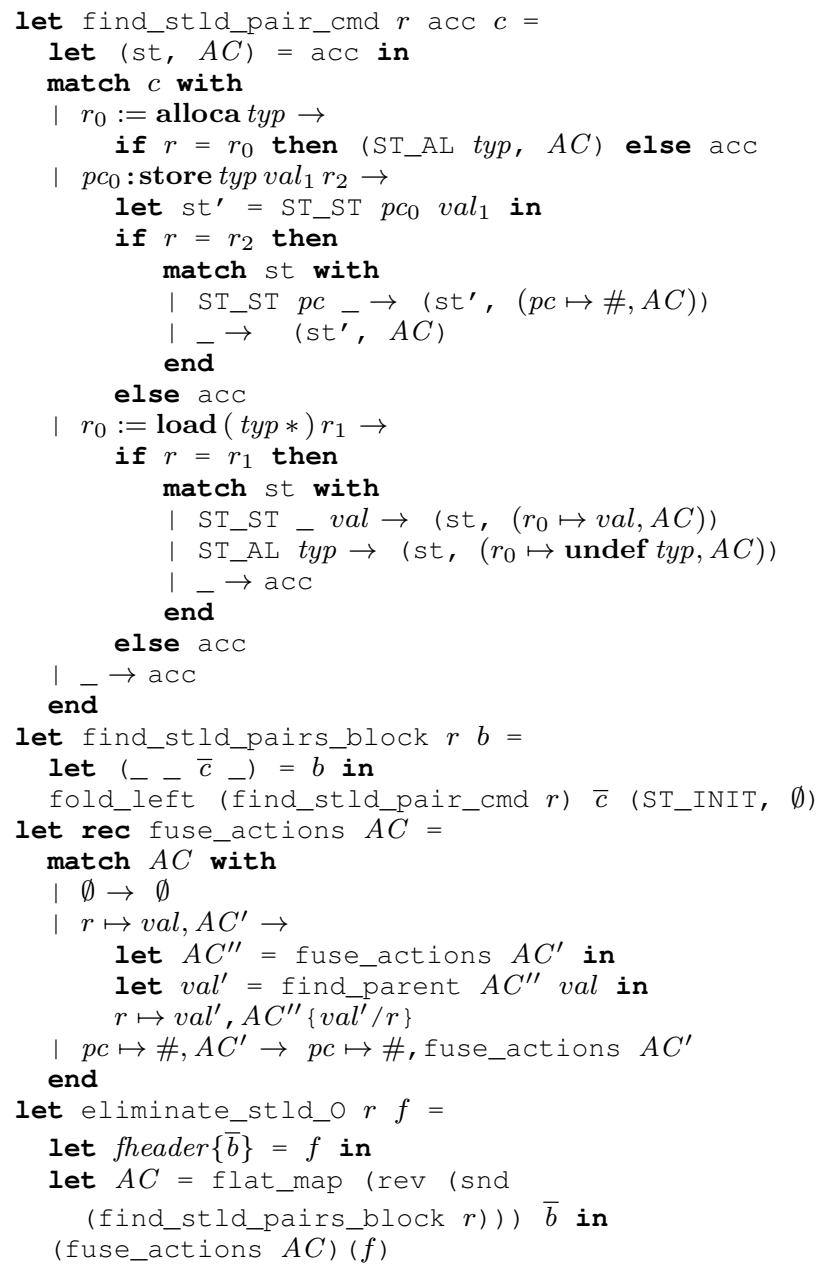

Figure 13. eliminate_stld of vmem 2 reg- 0

eliminate_stld_o must fuse the initial actions before transforming $f$. Let values be vertices, and elements in actions be edges. A list of actions found from a well-formed function forms a forest because SSA ensures acyclicity of def/use chains. fuse_actions fuses the forest to be a forest with depth one: each non-root node in a tree of the original forest maps to the root of the tree. In fuse_actions, find_parent $A C$ val returns the parent of $\mathrm{val}$ in the forest formed by $A C$ if $\mathrm{val}$ has a parent; otherwise returns val; $A C\{\mathrm{val} / r\}$ substitutes $r$ in the codomain of $A C$ by val. Suppose that eliminate_st ld_o finds a list of actions: $r_{4} \mapsto r_{3}, r_{5} \mapsto r_{4}, r_{2} \mapsto r_{1}, r_{3} \mapsto r_{2}, r_{6} \mapsto r_{3}$, []. fuse_actions returns $r_{4} \mapsto r_{1}, r_{5} \mapsto r_{1}, r_{2} \mapsto r_{1}, r_{3} \mapsto$ $r_{1}, r_{6} \mapsto r_{1},[]$.

The interesting part for the correctness of vmem2reg-O is showing that the fused pass produces the same output as the pipelined transformations:

LEMMA 29 (eliminate_stld_O is correct). If prog $\vdash f$, then eliminate_stld $r f=$ eliminate_stld_o $r f$.

By Lemma 29 and Theorem 30, we have that

THEOREM 30 (vmem2reg-O is correct). If $f^{\prime}=$ vmem2reg-O $f$ and $\vdash \operatorname{prog}$, then $\vdash \operatorname{prog}\left\{f^{\prime} / f\right\}$ and $\operatorname{prog} \supseteq \operatorname{prog}\left\{f^{\prime} / f\right\}$.

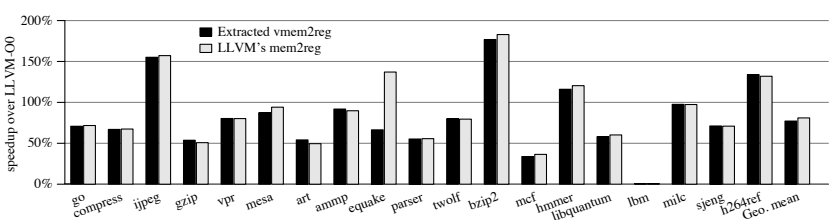

Figure 14. Execution speedup over LLVM - O0 for both the extracted vmem2 reg and LLVM's original mem2 reg pass.

\section{Discussion and Evaluation}

Coq Development Our Coq development for SSA optimizations consists of approximately 838 lines of algorithm implementations and 50k lines of (not particularly automated) correctness proof scripts and supporting infrastructure. We expect that much of these proofs can be reused for other SSA-based optimizations-that is the reason why we chose the pipeline of micro transformations structure. The development relies on about a dozen axioms, almost all of which define either the initial state of the machine (i.e., where in memory functions and globals are stored) or the behavior of external function calls. One axiom asserts that memory alignment is a power of two, which is not necessary for LLVM programs in general, but is true of almost all real-world platforms.

Extracted vmem2 reg and experimental methodology We used the Coq extraction mechanism to obtain a certified implementation of the vmem2reg optimization directly from the Coq sources. mem2reg is the first optimization pass applied by LLVM $^{4}$, so we tested the efficacy of the extracted implementation on LLVM IR bitcode generated directly from $\mathrm{C}$ source code using the clang compiler. At this stage, the LLVM bitcode is unoptimized and in "trivial" SSA form. To prevent the impact of this optimization pass from being masked by subsequent optimizations, we apply either LLVM's mem 2 reg or the extracted vmem 2 reg to the unoptimized LLVM bitcode and then immediately invoke the back-end code generator. We evaluate the performance of the resultant code on a $2.66 \mathrm{GHz}$ Intel Core 2 processor running benchmarks selected from the SPEC CPU benchmark suite that consist of over 336k lines of C source code in total.

Figure 14 reports the execution time speedups (larger is better) over a LLVM's-O0 compilation baseline for various benchmarks. The left bar of each group shows the speedup of the extracted vmem 2 reg, which provides an average speedup of $77 \%$ over the baseline. The right bar of each group is the benefit provided by LLVM's mem 2 reg, which provides $81 \%$ on average; vmem 2 reg captures much of the benefit of the LLVM's mem2reg.

Comparing vmem2reg and mem2reg The vmem2reg pass differs from LLVM's mem2reg in a few ways. First, mem2reg promotes allocas used by LLVM's intrinsics, while vmem 2 reg conservatively considers such allocas to potentially escape, and so does not promote them. We determined that such intrinsics (used by LLVM to annotate the liveness of variable definitions) lead to almost all the difference in performance in the equake benchmark. Second, although vmem 2 reg deletes most unused $\phi$-nodes, it does not aggressively remove them and, therefore, does not generate fully pruned SSA as mem 2 reg does. However, our results show that this does not impose a significant difference in performance.

Compilation time The focus of this work is compiler correctness, not compilation time. Although the code generated by vmem 2 reg is comparable to that of mem 2 reg, their compilation times differ, for two reasons. First, the extracted OCaml programs use purely functional data structures that impose $O(\log n)$ overhead compared to the efficient imperative hash tables available in $\mathrm{C}++$. Second, the

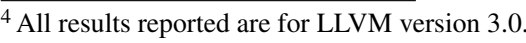


pessimistic $\phi$-node placement algorithm introduces unnecessary $\phi$ nodes. In our future work, we plan to use the minimal $\phi$-node placement algorithm in vmem2reg, whose correctness is (by design) independent of the proofs presented in this paper. Moreover, unlike "all or nothing" translations, vmem2reg maintains the SSA invariants at each pass and can thus be applied incrementally to boost performance. In practice, should compilation time be an issue, it is always possible to use mem 2 reg for development and vmem2reg to ensure correctness for a final release.

\section{Related Work and Conclusions}

Verifying the correctness of compiler transformations is an active research area with a sizable amount of literature. We focus here on the work relevant to SSA-based optimizations.

CompCertSSA CompCertSSA [4] improves the CompCert compiler with a verified SSA-based middle-end and a GVN optimization pass. CompCertSSA verified a translation validator for an SSA construction algorithm that takes imperative variables to variables in a pruned SSA form. In contrast, our work fully verifies the SSA construction pass vmem2 reg for LLVM directly. A bug in the CompCertSSA compiler will cause the validator to abort the compilation, whereas verifying the compiler rules out such a possibility. More pragmatically, translation validation is harder to apply in the context of LLVM, because the compiler infrastructure was not created with verification in mind. For example, the CompCertSSA translations maintain a close mapping between source and target variable names so that simulation can be checked by simple erasure; this is not feasible in the LLVM framework. The CompCertSSA project reports performance measurements of only small benchmarks totaling about $6 \mathrm{k}$ lines, whereas we have tested our pass on 336k lines, including larger programs.

Unsurprisingly, the CompCertSSA and Vellvm proofs share some similarities. For example, CompCertSSA's GVN proof uses an invariant similar to the one in our Theorem 8 and Lemma 12. However, the LLVM's strategy of promoting allocas means that our proofs need a combination of both SSA and aliasing properties to prove correctness. Moreover, our proof technique of pipelining "micro" transformations is novel, and should be broadly applicable.

Other related work In less closely related work, Mansky et al. designed an Isabelle/HOL framework that uses control-flow graph rewrites to transform programs and uses temporal logic and modelchecking to specify and prove the correctness of program transformations [11]. They verified an SSA construction algorithm in the framework. Other researchers have formalized specific SSAbased optimizations by using SSA forms with different styles of semantics: an informal semantics that describes the intuitive idea of the SSA form [7]; an operational semantics based on a matrix representation of $\phi$ nodes [19]; a data-flow semantics based term graphs using the Isabelle/HOL proof assistant [5]. Matsuno et al. defined a type system equivalent to the SSA form and proved that dead code elimination and common subexpression elimination preserve types [12]. There are also conversions between the programs in SSA form and functional programs [2,8].

Conclusion We have presented a proof technique for formally verifying SSA-based compiler optimizations. Using the Coq proof assistant, we fully mechanized the proof technique and the correctness of several micro optimizations. For the full LLVM IR semantics in Vellvm, we have formalized and implemented an extractable SSA optimization pass vmem2reg that is an easier-to-prove variant of LLVM's mem2reg pass but that delivers most of its benefits.

Acknowledgments We would like to thank Delphine Demange, Vivien Durey, and Dmitri Garbuzov for their comments and suggestions about this work.

\section{References}

[1] Static Single Assignment Book, 2012. Working draft available at http://ssabook.gforge.inria.fr/latest/book.pdf.

[2] A. W. Appel. SSA is functional programming. SIGPLAN Not., 33(4): 17-20, April 1998. ISSN 0362-1340.

[3] J. Aycock and N. Horspool. Simple generation of static single assignment form. In $C C, 2000$.

[4] G. Barthe, D. Demange, and D. Pichardie. A formally verified SSAbased middle-end - Static Single Assignment meets CompCert. In ESOP, 2012.

[5] J. O. Blech, S. Glesner, J. Leitner, and S. Mülling. Optimizing code generation from SSA form: A comparison between two formal correctness proofs in Isabelle/HOL. Electron. Notes Theor. Comput. Sci., 141(2):33-51, 2005.

[6] The Coq Proof Assistant Reference Manual (Version 8.3pl1). The Coq Development Team, 2011.

[7] R. Cytron, J. Ferrante, B. K. Rosen, M. N. Wegman, and F. K. Zadeck. Efficiently computing static single assignment form and the control dependence graph. TOPLAS, 13:451-490, 1991.

[8] R. A. Kelsey. A correspondence between continuation passing style and static single assignment form. In $I R$, number 3, 1995.

[9] X. Leroy. A formally verified compiler back-end. Journal of Automated Reasoning, 43(4):363-446, December 2009. ISSN 0168-7433.

[10] The LLVM Reference Manual (Version 3.0). The LLVM Development Team, 2011. http://1lvm.org/releases/3.0/docs/ LangRef. html.

[11] W. Mansky and E. L. Gunter. A framework for formal verification of compiler optimizations. In ITP, 2010.

[12] Y. Matsuno and A. Ohori. A type system equivalent to static single assignment. In PPDP, 2006.

[13] V. S. Menon, N. Glew, B. R. Murphy, A. McCreight, T. Shpeisman, A. Adl-Tabatabai, and L. Petersen. A verifiable SSA program representation for aggressive compiler optimization. In POPL, 2006.

[14] S. S. Muchnick. Advanced compiler design and implementation. Morgan Kaufmann Publishers Inc., San Francisco, CA, USA, 1997. ISBN 1-55860-320-4.

[15] V. C. Sreedhar and G. R. Gao. A linear time algorithm for placing $\phi$-nodes. In POPL, 1995.

[16] J.-B. Tristan and X. Leroy. Formal verification of translation validators: a case study on instruction scheduling optimizations. In $P O P L$, 2008.

[17] J.-B. Tristan and X. Leroy. Verified validation of lazy code motion. In PLDI, 2009.

[18] J. B. Tristan and X. Leroy. A simple, verified validator for software pipelining. In POPL, 2010.

[19] B. Yakobowski. Étude sémantique d'un langage intermédiaire de type Static Single Assignment. Rapport de dea (Master's thesis), ENS Cachan and INRIA Rocquencourt, Sept. 2004.

[20] X. Yang, Y. Chen, E. Eide, and J. Regehr. Finding and understanding bugs in C compilers. In PLDI, 2011.

[21] J. Zhao and S. Zdancewic. Mechanized verification of computing dominators for formalizing compilers. In CPP, 2012.

[22] J. Zhao, S. Nagarakatte, M. M. K. Martin, and S. Zdancewic. Formalizing the LLVM intermediate representation for verified program transformations. In POPL, 2012.

This research was funded in part by the U.S. Government. The views and conclusions contained in this document are those of the authors and should not be interpreted as representing the official policies, either expressed or implied, of the U.S. Government. This research was funded in part by DARPA contract HR0011-10-9-0008 and ONR award N000141110596. This material is based upon work supported by the National Science Foundation under Grant No. CNS-1116682, CCF-1065166, and CCF-0810947. Any opinions, findings, and conclusions or recommendations expressed in this material are those of the author(s) and do not necessarily reflect the views of the National Science Foundation. 